\title{
Editorial: Uncertainty Visualization and Decision Making
}

\author{
Roberto Theron ${ }^{1 *}$ and Lace M. Padilla ${ }^{2}$ \\ ${ }^{1}$ VisUSAL-GRIAL, Department of Computer Science and Automation, University of Salamanca, Salamanca, Spain, ${ }^{2}$ Department \\ of Psychology, University of California, Merced, Merced, CA, United States
}

Keywords: uncertainty visualization, decision making, uncertainty, visualization, data analysis

\section{Editorial on the Research Topic}

\section{Uncertainty Visualization and Decision Making}

The conceptualization of uncertainty-and related concepts, such as ambiguity, ignorance, or risk-has proven to be an elusive task (Lipshitz and Strauss, 1997; Therón Sánchez et al., 2019). Different domains have diverse understandings of these concepts. As such, critical factors for the success of methodologies and tools to facilitate understanding uncertainty include the decision maker's previous training, level of expertise, and the scenario's aspects.

After the introduction of the range bar (Spear, 1952) by Mary Eleanor Spear and the ubiquitous box plots (Tukey et al., 1977) by John Tukey, the use of graphical representations to help analysts understand the inherent uncertainty in the scientific process is well established in many fields. Despite the popularity of these plots and the many variations and novel visualization techniques that researchers have designed in the last decades, several questions remain unanswered that drive the recent surge of uncertainty visualization research. Adding to the breadth of work on this topic are many powerful visualization techniques that researchers later modified, but designers did not initially intend to convey uncertainty-e.g., parallel coordinate plots (Dasgupta et al., 2012). Adapting existing techniques is often challenging (Bonneau et al., 2014), which researchers approached using various methods to map uncertainty (Pang et al., 1997).

Notably, the fact that a visualization incorporates uncertainty information does not necessarily lead to more informed decision-making. The contribution of uncertainty visualizations needs to be assessed based on performance and user experience together with interpretation and decision quality (Hullman et al., 2018).

We intended this Research Topic to deepen the understanding of the way people make decisions based on the processing of information on uncertainty provided through different forms of visualization. Thus, it contributes to the much-needed reflection on various factors in humancomputer interaction that can drive visualization towards more efficient and proven ways that help improve decision-making in increasingly complex scenarios.

The articles in this Research Topic exemplify exciting new approaches in the uncertainty visualization research sub-discipline. Beyond the intention of communicating uncertainty in the design of the different visualization techniques, the actual capacities of people to interpret the information and how they reason about the associated phenomena are considered and exploited. This collection of articles presents ongoing research that investigates the benefits and limitations brought by uncertainty visualization to support effective reasoning about data, considering specific scenarios and the characteristics of their particular users.

One of the most popular methodologies to support decision making under uncertainty is Bayesian analysis. Taka et al. propose a method to transform Bayesian probabilistic models into interactive graphical representations, achieving an instrument with better interpretability, closer to the human users. 
Supporting decision making with map displays has a long tradition. Korporaal et al. studied how uncertainty visualization may affect decision-makers in time-critical and complex spacetime decision contexts.

In a related study, Parmar et al. investigated the benefits of communicating cumulative risk as a way to convey uncertainty. The authors relate the mistakes made by pilots in dangerous meteorological operations with a misunderstanding of the real risks derived from specific conditions so that the adequate visual communication of this risk can favour decision-making.

Similarly, Lindell addresses this communication challenge, recognizing the fact that people can process the information present in hazard maps with different levels of accuracy, which results in the extraction of wrong or correct conclusions; the research focuses on trying to propose better ways of visualization that help to make more accurate inferences.

Improving hazard map comprehension is also the goal of Padilla et al. in a research work that considers situations where people have to deal with multiple hazards with various types of

\section{REFERENCES}

Bonneau, G.-P., Hege, H.-C., Johnson, C. R., Oliveira, M. M., Potter, K., Rheingans, P., et al. (2014). "Overview and State-Of-The-Art of Uncertainty Visualization," in Scientific Visualization (London: Springer), 3-27. doi:10.1007/978-1-44716497-5_1

Dasgupta, A., Chen, M., and Kosara, R. (2012). Conceptualizing Visual Uncertainty in Parallel Coordinates. Comput. Graph. Forum 31, 1015-1024. doi:10.1111/ j.1467-8659.2012.03094.x

Hullman, J., Qiao, X., Correll, M., Kale, A., and Kay, M. (2019). In Pursuit of Error: A Survey of Uncertainty Visualization Evaluation. IEEE Trans. Vis. Comput. Graphics 25, 903-913. doi:10.1109/tvcg.2018.2864889

Lipshitz, R., and Strauss, O. (1997). Coping with Uncertainty: A Naturalistic Decision-Making Analysis. Organizational Behav. Hum. Decis. Process. 69, 149-163. doi:10.1006/obhd.1997.2679

Pang, A. T., Wittenbrink, C. M., and Lodha, S. K. (1997). Approaches to Uncertainty Visualization. Vis. Comput. 13, 370-390. doi:10.1007/s003710050111

Sánchez, R. T., Santos, A. B., Vicente, R. S., and Gómez, A. L. (2019). Towards an Uncertainty-Aware Visualization in the Digital Humanities. Informatics 6, 31. doi:10.3390/informatics6030031 uncertainties. Through a reflection on three significant challenges related to the communication of uncertainty in multiple hazard scenarios, the authors pave the way for a more systematic approach that the research community may embrace.

The last two articles share the objective to shed light on how people reason about uncertainty with the aid of visualizations. Padilla et al. conducted two experiments to explore how participants utilize the quantitative and qualitative uncertainty conveyed by different visualization techniques. Finally, Joslyn et al. are interested in how non-expert end-users reason about uncertainty visualizations, providing a psychological explanation to the deterministic construal error-the tendency to interpret the conveyed uncertainty as deterministic-and offer solutions to mitigate it.

\section{AUTHOR CONTRIBUTIONS}

All authors listed have made a substantial, direct and intellectual contribution to the work, and approved it for publication.

Spear, M. E. (1952). Charting Statistics. New York: McGraw-Hill.

Tukey, J. W. (1977). Exploratory Data Analysis, Vol. 2. Reading, Mass: AddisonWesley.

Conflict of Interest: The authors declare that the research was conducted in the absence of any commercial or financial relationships that could be construed as a potential conflict of interest.

Publisher's Note: All claims expressed in this article are solely those of the authors and do not necessarily represent those of their affiliated organizations, or those of the publisher, the editors and the reviewers. Any product that may be evaluated in this article, or claim that may be made by its manufacturer, is not guaranteed or endorsed by the publisher.

Copyright (C) 2021 Theron and Padilla. This is an open-access article distributed under the terms of the Creative Commons Attribution License (CC BY). The use, distribution or reproduction in other forums is permitted, provided the original author(s) and the copyright owner(s) are credited and that the original publication in this journal is cited, in accordance with accepted academic practice. No use, distribution or reproduction is permitted which does not comply with these terms. 